Supporting Information

\title{
A Smart Superhydrophobic Surface with Restorable Microstructure and Self-healable Surface Chemistry
}

\author{
Shengyang Pan, Min Chen* and Limin Wu \\ Department of Materials Science and State Key Laboratory of Molecular Engineering of \\ Polymers, Advanced Coatings Research Center of Ministry of Education of China, Fudan \\ University, Shanghai 200433, China
}

Figure S1-S8

Supplementary Videos:

Movie S1

Movie S2

*Corresponding author, email: chenmin@ fudan.edu.cn 

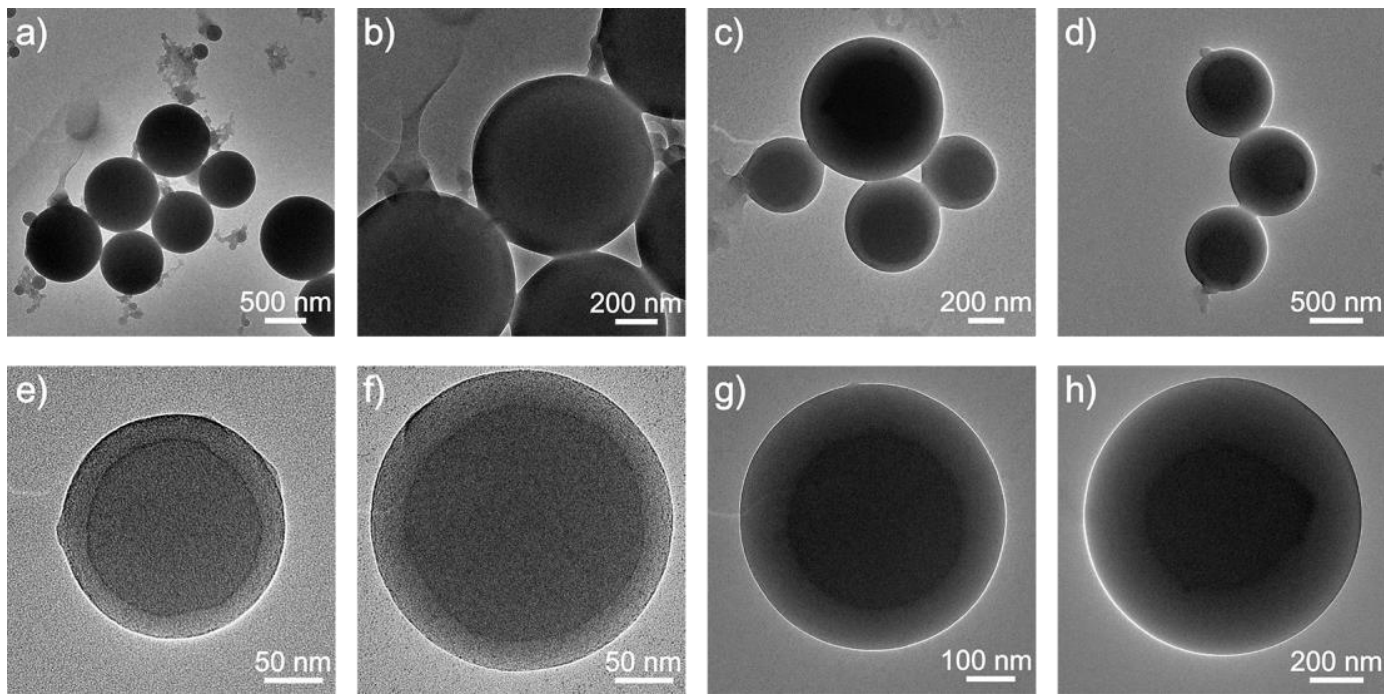

Figure S1. (a), (b) TEM images of NF-MS. (c)-(h) TEM images of F-MC, obvious core-shell structure of the F-MC with various particle sizes from $200 \mathrm{~nm}$ to $1 \mu \mathrm{m}$ could be observed. 
a)

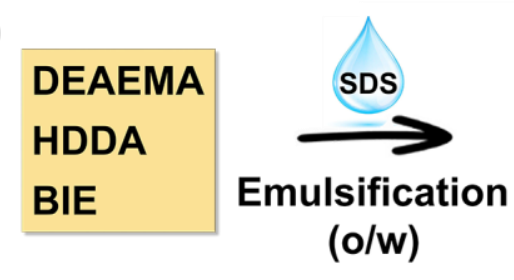

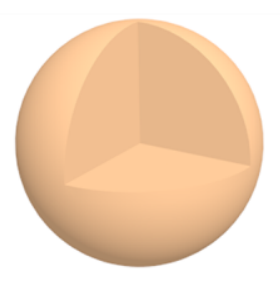

UV 365 nm
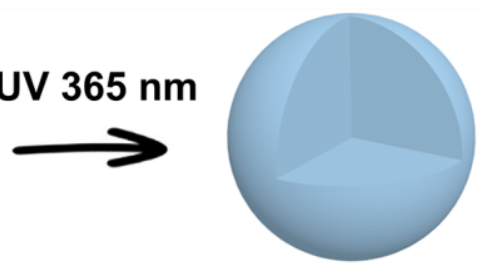

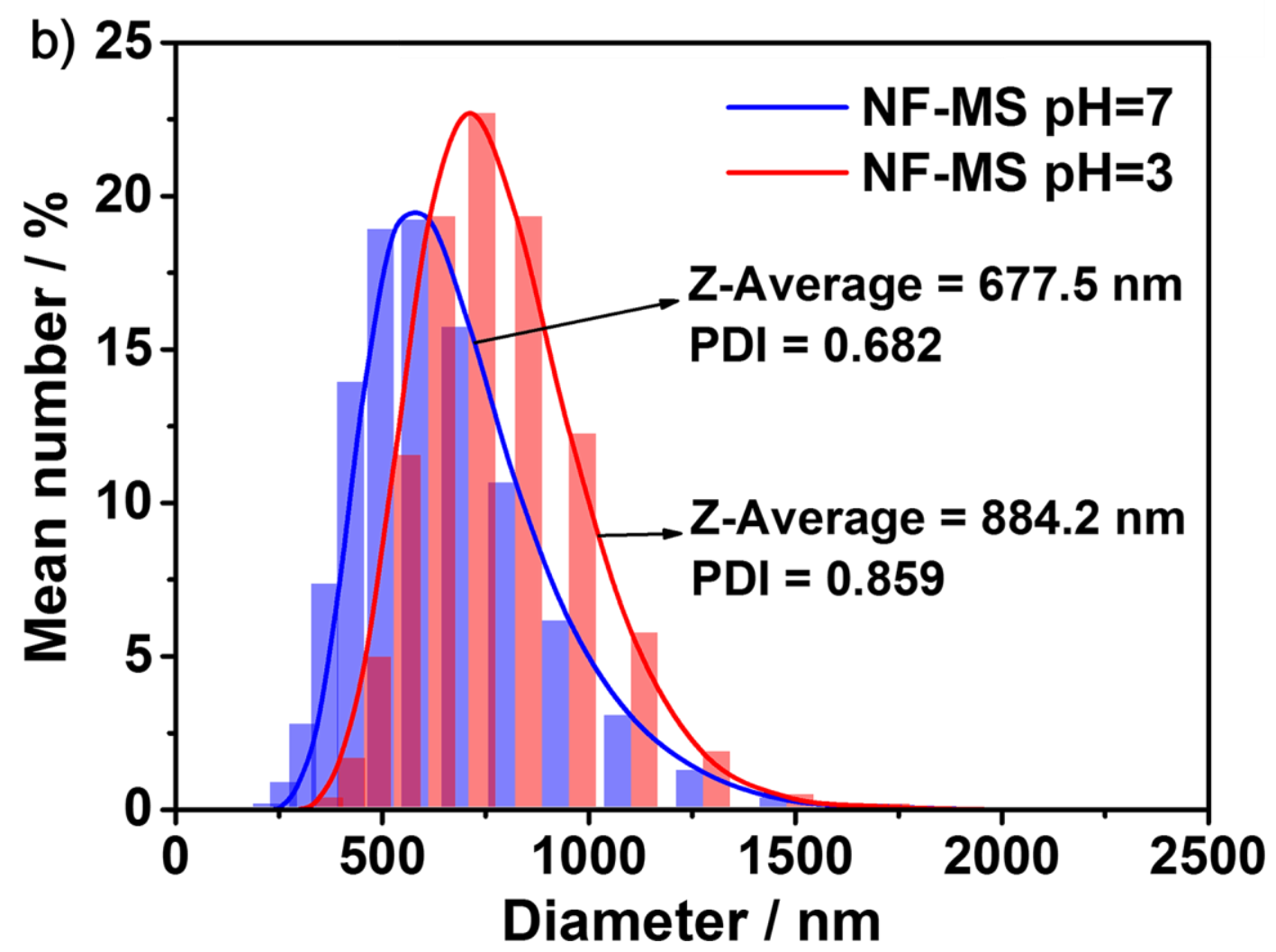

Figure S2. (a) Schematic illustration of the synthesis of NF-MS. (b) Change in particle size distribution of NF-MS before $(\mathrm{pH}=7)$ and after $(\mathrm{pH}=3)$ acidization measured by DLS. 

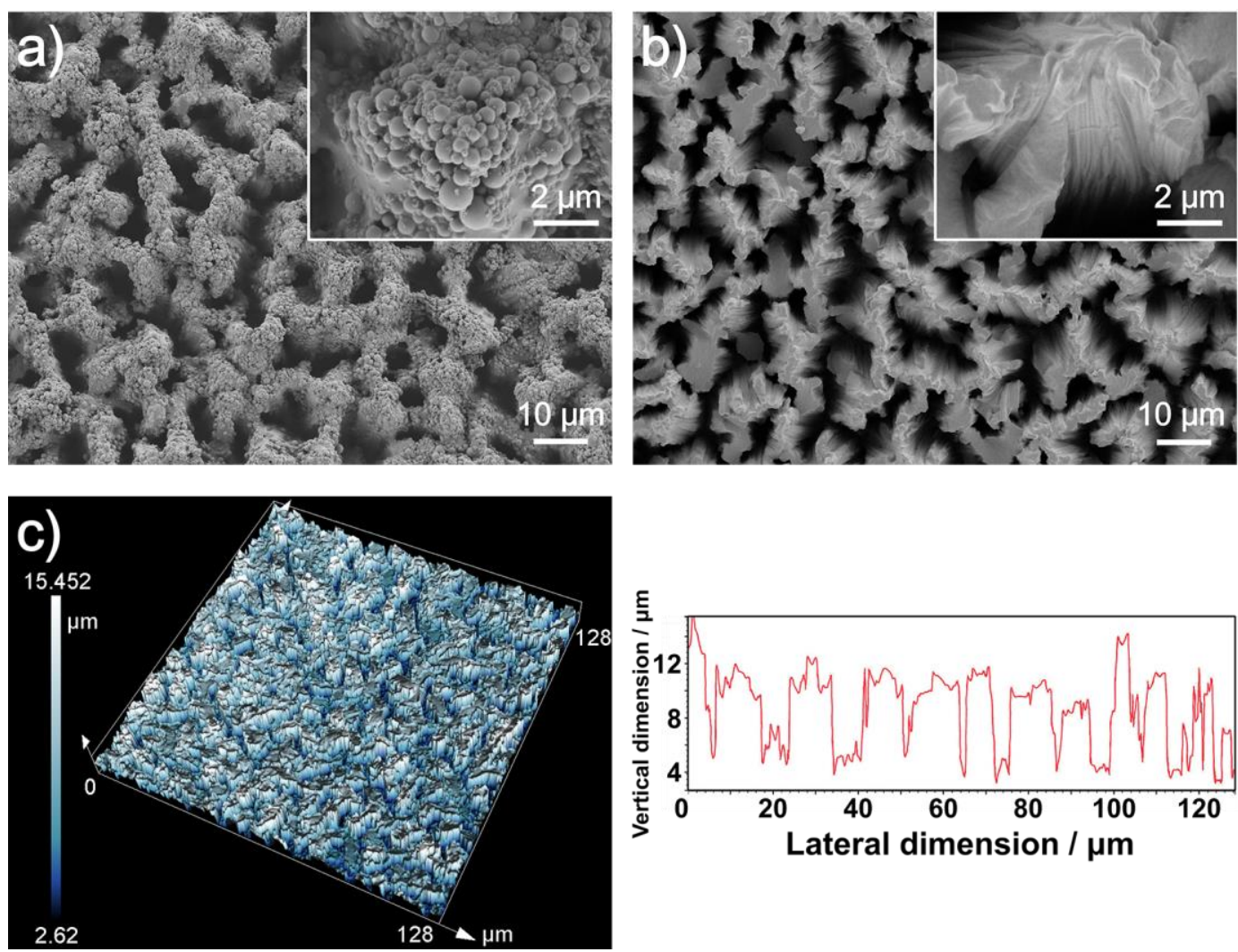

Figure S3. (a) SEM image of PDMS mould surface precoated by F-MC. (b) SEM image of PDMS mould after the demoulding process of shape memory epoxy resin. (c) 3D confocal image and its corresponding profile picture of PDMS mould fabricated using our previous method. 


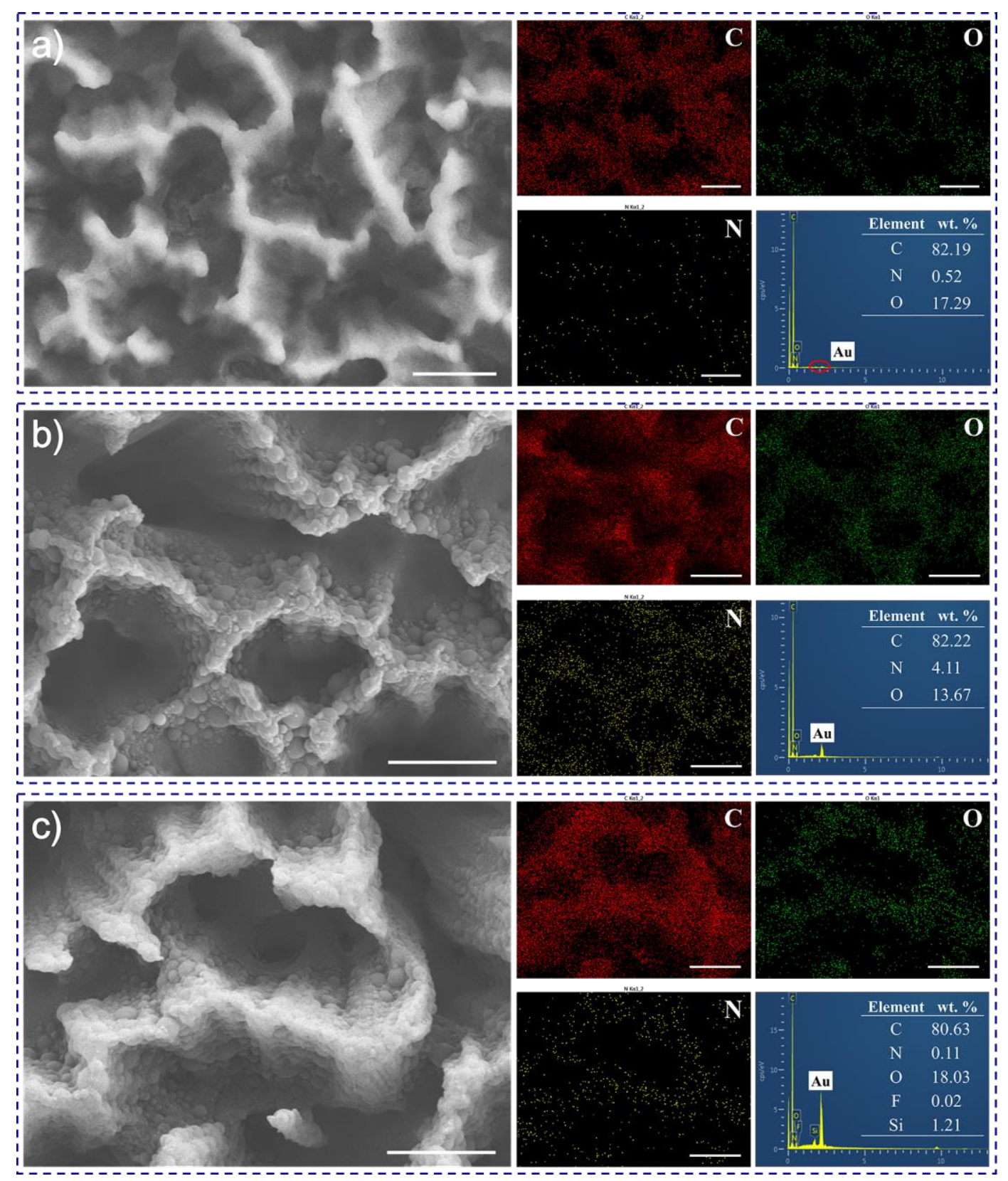

Figure S4. EDX mapping and spectrum of the (a) MP, (b) NF-MS-MP and (c) F-MC-MP surface. Scale bar $=5 \mu \mathrm{m}$. 

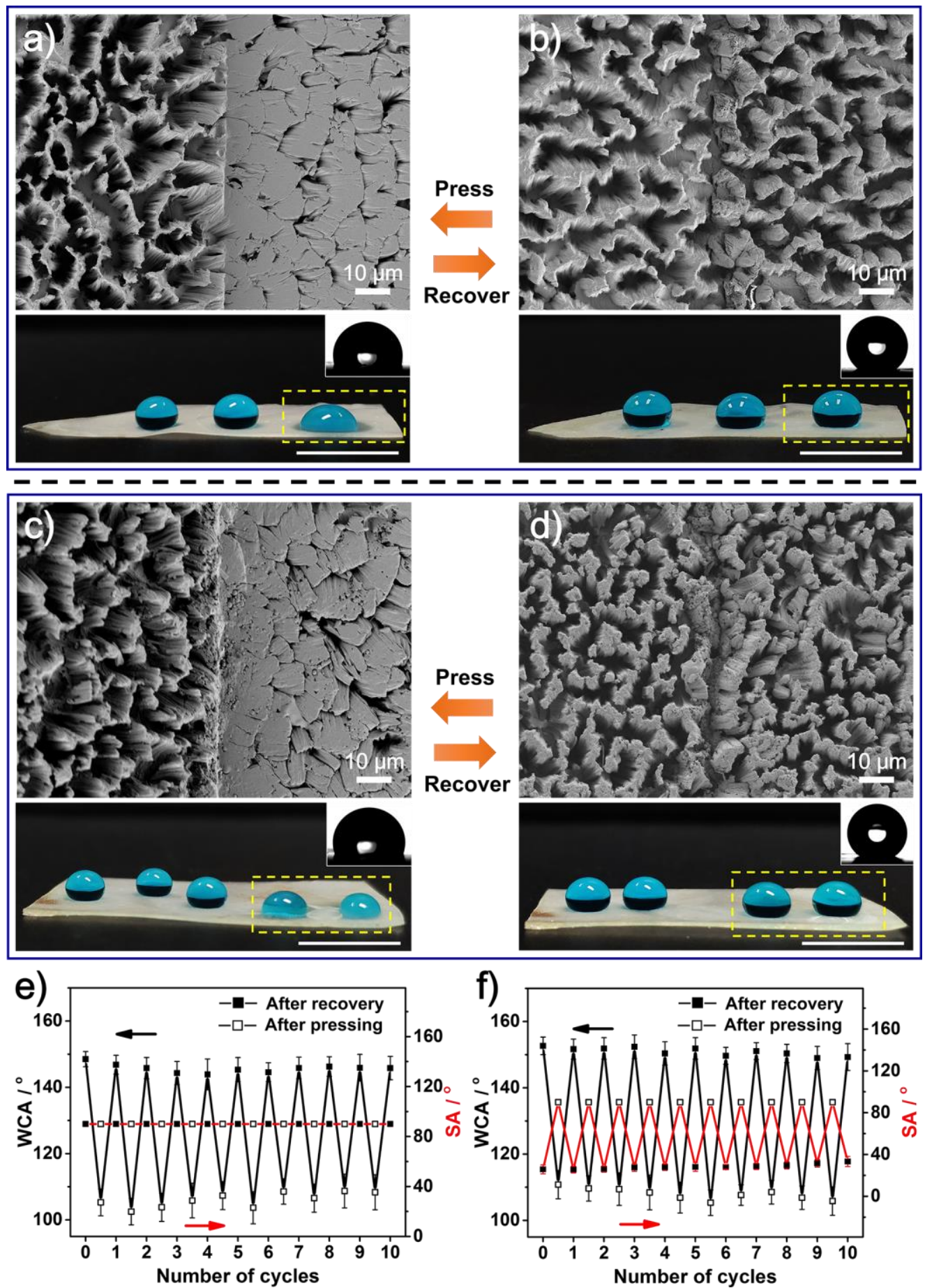

Figure S5. SEM image and optical image of MP surface (a) and NF-MS-MP surface (c) after localized pressing (collapsed micropillars on the right half), water droplets pin on the pressed area with WCA of $107^{\circ}$ and $112^{\circ}$, respectively. SEM image and optical image of MP surface (b) and NF-MS-MP surface (d) after recovery, water droplets sit onto the recovered area in spherical shape (on the right) with WCA of $149^{\circ}$ and $151^{\circ}$, respectively. (e), (f) The variation of WCA and SA of MP and NF-MS-MP after several consecutive pressing/recovery processes, respectively. 

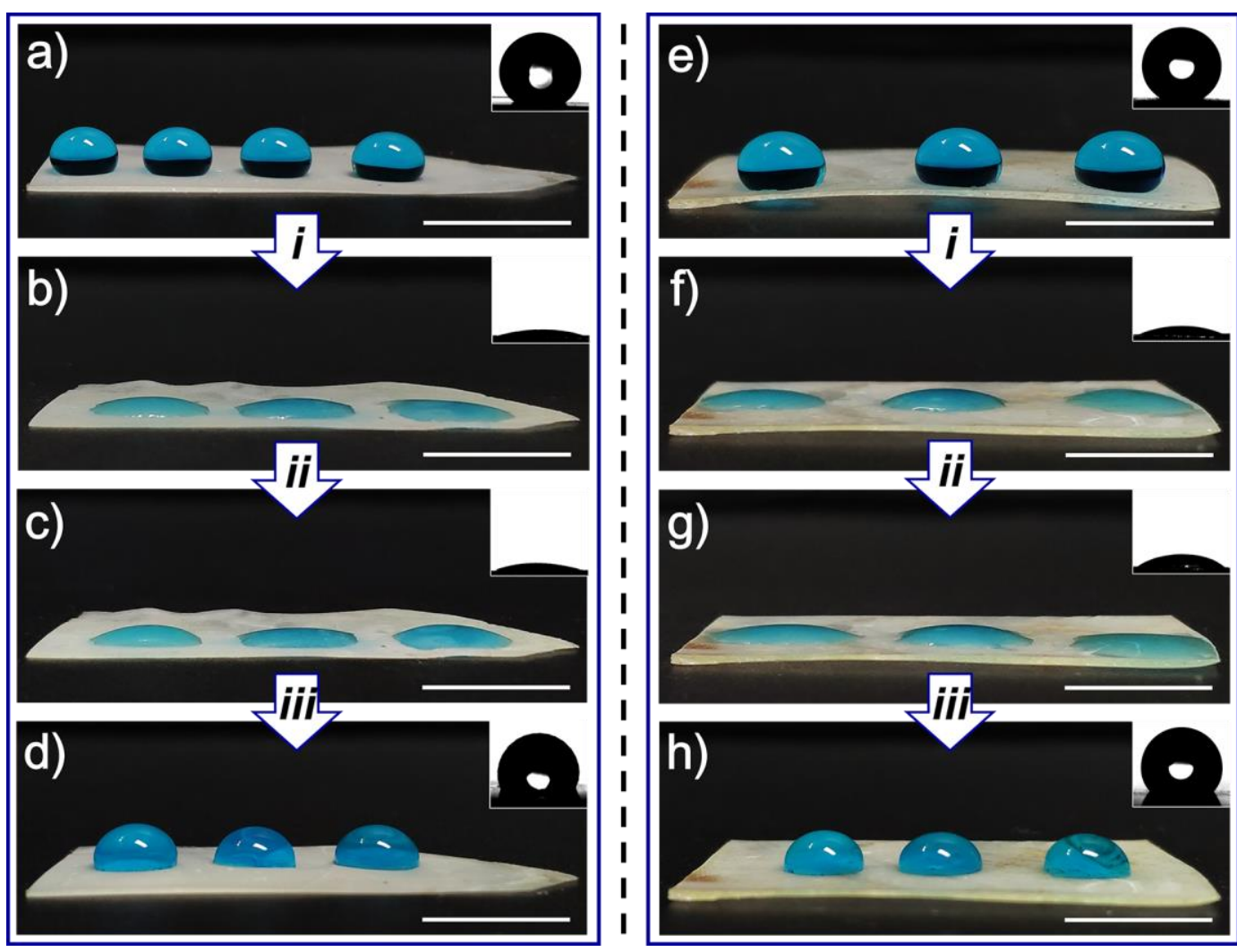

Figure S6. (a), (e) The as-prepared MP and NF-MS-MP surface with static WCA of $148^{\circ}$ and $152^{\circ}$, respectively. (b), (f) The MP and NF-MS-MP surface after oxygen plasma treatment (Process i) with WCA decreased to $21^{\circ}$ and $25^{\circ}$, respectively. (c), (g) The MP and NF-MS-MP surface placed in oven at $50{ }^{\circ} \mathrm{C}$ for $10 \mathrm{~min}$ (Process ii) after oxygen plasma treatment, the WCA changed little of $26^{\circ}$ and $32^{\circ}$ respectively, compared to (b) and (f). (d), (h) The MP and NF-MS-MP surface was subsequently heated up to $120{ }^{\circ} \mathrm{C}$ in an oven for $5 \min$ (Process iii), the static WCA increased to $125^{\circ}$ and $132^{\circ}$ respectively. 

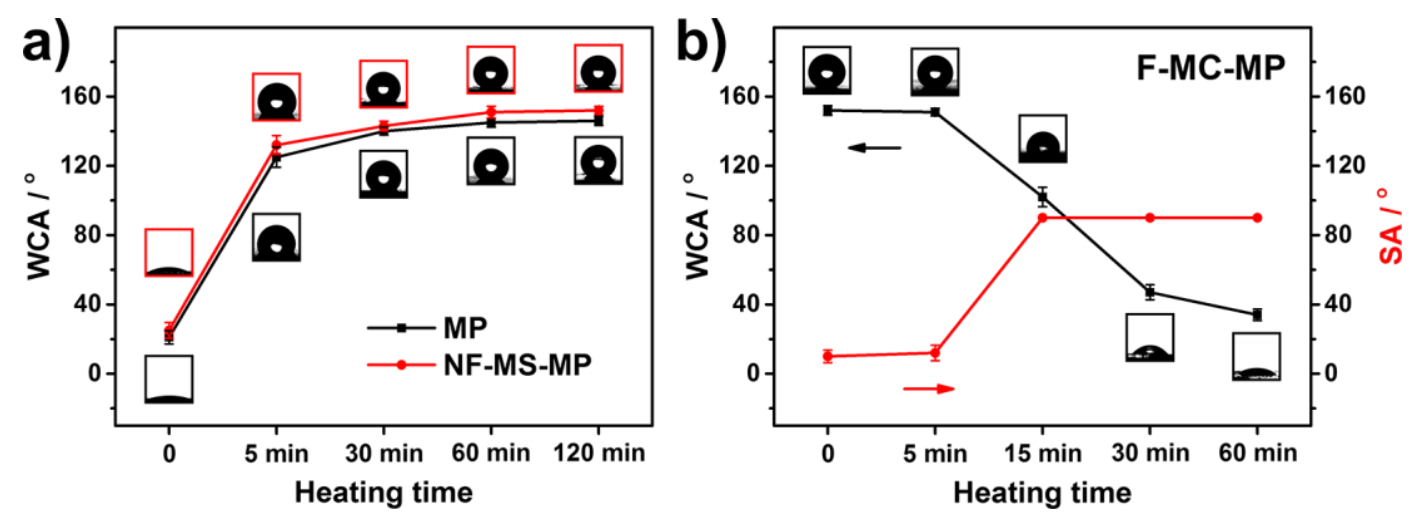

Figure S7. (a) Variation of WCA as a function of heating time under $120^{\circ} \mathrm{C}$ after oxygen plasma treatment of MP and NF-MS-MP surfaces. (b) After previous heating at $105{ }^{\circ} \mathrm{C}$ for certain amount of time, the surface chemistry of F-MC-MP was then destroyed and recovered in acid. The variation of WCA and SA of samples after acid immersion reflects the change of healing ability of surface chemistry caused by previous heating process. 

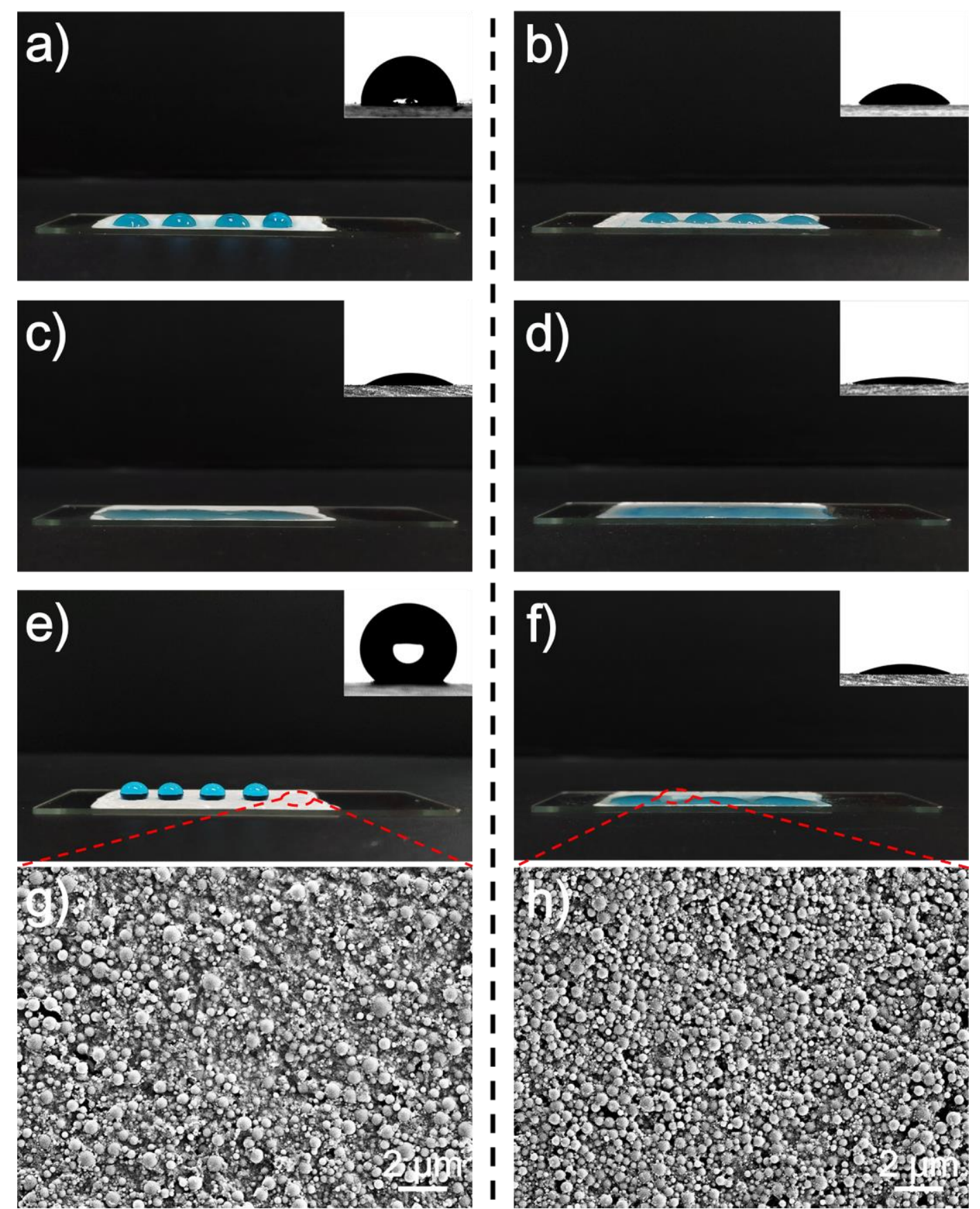

Figure S8. (a)-(f) Optical images of water droplets $(50 \mu \mathrm{L}$, dyed in blue) spreading on the surface of F-MC and NF-MS coated glass substrate. (a), (b) Original F-MC and NF-MS coated surfaces respectively. Insets: WCA of $90^{\circ}$ and $46^{\circ}$ for F-MC and NF-MS surfaces respectively. (c), (d) Surfaces after oxygen plasma treatment. Insets: WCA of $24^{\circ}$ and $13^{\circ}$ respectively. (e), (f) Surfaces after heating at $120^{\circ} \mathrm{C}$. Insets: WCA of $141^{\circ}$ and $21^{\circ}$ respectively. (g) and (h) Surface morphologies of F-MC and NF-MS coated glass substrate observed by FESEM. 